Supplementary Information for:

\title{
A Macrocyclic 1,4-Diketone Enables the Synthesis of a p-Phenylene Ring that is More Strained than a Monomer Unit of [4]Cycloparaphenylene
}

\author{
Nirmal K. Mitra, Hector H. Corzo, and Bradley L. Merner* \\ Department of Chemistry and Biochemistry, Auburn University, Auburn, AL, 36849
}

\section{TABLE OF CONTENTS}

1. Scheme SI-1: Structures/compounds not numbered in the manuscript that appear in the SI

2. General experimental conditions, procedures, and characterization data

3. ${ }^{1} \mathrm{H}$ and ${ }^{13} \mathrm{C}$ NMR spectra

4. DFT Cartesian coordinates for $\mathbf{9}, \mathbf{1 2}$, and $\mathbf{1 3}$

1.

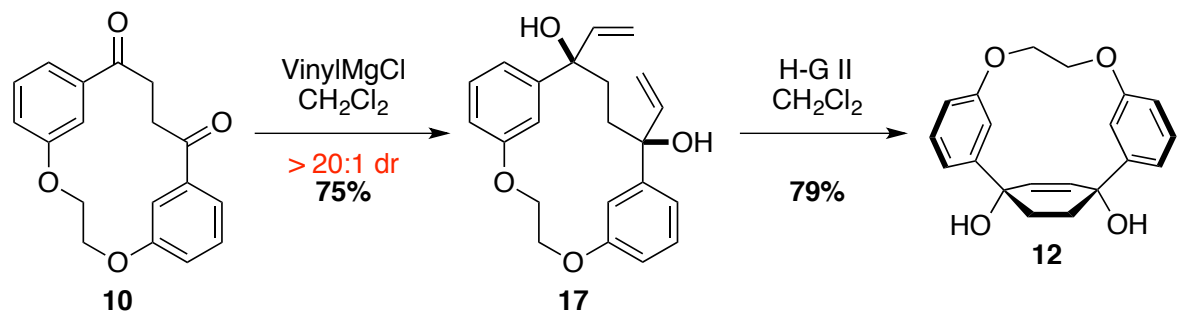

Scheme SI-1: Structures/compounds not numbered in the manuscript that appear in the SI

\section{General Experimental Conditions}

All reactions were run in flame or oven-dried $\left(120^{\circ} \mathrm{C}\right)$ glassware and cooled under a positive pressure of ultra high pure nitrogen or argon gas. All chemicals were used as received from commercial sources, unless otherwise stated. Anhydrous reaction solvents were purified and dried by passing HPLC grade solvents through activated columns of alumina (Glass Contour SDS). All solvents used for chromatographic separations were HPLC grade (hexanes, ethyl acetate, dichloromethane, chloroform, methanol, and acetone). Chromatographic separations were preformed using flash chromatography, as originally reported by Still and co-workers, on silica gel 60 (particle size 43-60 $\mu \mathrm{m}$ ), and all chromatography conditions have been reported as height $\times$ diameter in centimeters. Reaction progress was monitored by thin layer chromatography (TLC), on glass-backed silica gel plates $(\mathrm{pH}=$ 7.0). TLC plates were visualized using a handheld UV lamp (254 nm) and stained using an aqueous ceric ammonium molybdate (CAM) solution. Plates were dipped, wiped clean, and heated from the back of the plate. ${ }^{1} \mathrm{H}$ and ${ }^{13} \mathrm{C}$ nuclear magnetic resonance (NMR) spectra were recorded at 400 or 600 
$\mathrm{MHz}$, calibrated using residual undeuterated solvent as an internal reference $\left(\mathrm{CHCl}_{3}, \delta 7.27\right.$ and 77.2 ppm), reported in parts per million relative to trimethylsilane (TMS, $\delta 0.00 \mathrm{ppm}$ ), and presented as follows: chemical shift $(\delta, \mathrm{ppm})$, multiplicity $(\mathrm{s}=$ singlet, br $\mathrm{s}=$ broad singlet, $\mathrm{d}=\mathrm{doublet}, \mathrm{dd}=\mathrm{doublet}$ of doublets, $\mathrm{ddd}=$ doublet of doublet of doublets, $\mathrm{t}=$ triplet, $\mathrm{m}=$ multiplet $)$, coupling constants $(J, \mathrm{~Hz})$. High-resolution mass spectrometric (HRMS) data were obtained using a quadrupole time-of-flight (QTOF) spectrometer and electrospray ionization (ESI).

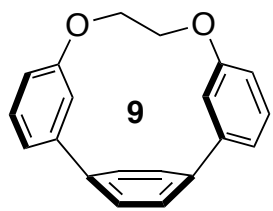

1,4-dioxa(3,3")[4]p-terphenylophane (9): A solution of n-butyllithium in hexanes (2.53 $\mathrm{M}, 0.80 \mathrm{~mL}, 2.0 \mathrm{mmol}$ ) was added dropwise to a stirred solution of diisopropylamine $(0.50 \mathrm{~mL}, 3.6 \mathrm{mmol})$ in toluene $(10 \mathrm{~mL})$ at $0{ }^{\circ} \mathrm{C}$. After $30 \mathrm{~min}$. , a solution of $15(0.024$ $\mathrm{g}$, $0.066 \mathrm{mmol})$ in toluene $(2.0 \mathrm{~mL})$ was added at $0{ }^{\circ} \mathrm{C}$. After $1 \mathrm{~h}$, the reaction was quenched with water $(20 \mathrm{~mL})$, the layers were separated, and the aqueous phase was extracted with dichloromethane $(2 \times 10$ $\mathrm{mL})$. The combined organic extracts were washed with brine $(30 \mathrm{~mL})$, dried over anhydrous $\mathrm{MgSO}_{4}$ and concentrated under reduced pressure. The residue was purified by flash chromatograph $(4 \times 0.6$ $\mathrm{cm}, 1: 1$ dichloromethane/hexanes) to afford 9 as a white solid $(0.012 \mathrm{~g}, 63 \%): R_{f}=0.42(2: 3$ dichloromethane/hexane; ${ }^{1} \mathrm{H}$ NMR (400 MHz, $\left.\mathrm{CDCl}_{3}\right) \delta 7.43$ (br s, 4H), 7.31-7.26 (m, 2H), 7.24-7.21 (m, 2H), $6.70(\mathrm{ddd}, J=8.2,2.9,1.0 \mathrm{~Hz}, 2 \mathrm{H}), 5.29(\mathrm{dd}, J=3.0,1.4 \mathrm{~Hz}, 2 \mathrm{H}), 4.20-3.76(\mathrm{~m}, 4 \mathrm{H}) ;{ }^{13} \mathrm{C}$ NMR $(101$ $\left.\mathrm{MHz}_{2} \mathrm{CDCl}_{3}\right) \delta 156.25,145.57,145.19,130.59,117.85,115.69,115.63,62.34$ (Only 8 of 9 signals are observed - $\delta 130.59$ corresponds to 2 carbons); HRMS (EI) calculated for $\mathrm{C}_{20} \mathrm{H}_{16} \mathrm{O}_{2}\left(\mathrm{M}^{+}\right) \mathrm{m} / z=288.1150$, found 288.1158

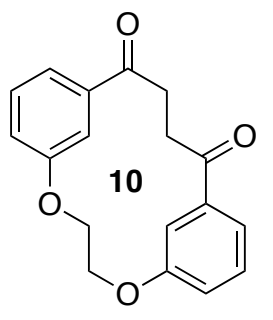

1,4-diketone 10: Vinylmagnesium chloride (1.6 M in THF, $7.3 \mathrm{~mL}, 12 \mathrm{mmol}$ ) was added to a stirred $0{ }^{\circ} \mathrm{C}$ solution of dialdehyde $11(1.25 \mathrm{~g}, 4.63 \mathrm{mmol})$ in $\mathrm{CH}_{2} \mathrm{Cl}_{2}$ (35 $\mathrm{mL})$. After $30 \mathrm{~min}$. , the reaction mixture was poured into water $(100 \mathrm{~mL})$ and further diluted with $1 \mathrm{M} \mathrm{HCl}(50 \mathrm{~mL})$. The resulting mixture was extracted with dichloromethane $(3 \times 40 \mathrm{~mL})$. The combined organic extracts were washed with a saturated solution of $\mathrm{NaHCO}_{3}(50 \mathrm{~mL})$ and brine $(50 \mathrm{~mL})$, dried over anhydrous $\mathrm{Na}_{2} \mathrm{SO} 4$, and filtered. The resulting solution was diluted with dichloromethane $(320 \mathrm{~mL})$, heated to $40{ }^{\circ} \mathrm{C}$, followed by the addition of the Hoveyda-Grubbs second-generation catalyst $(0.080 \mathrm{~g}, 0.13 \mathrm{mmol})$. After $4 \mathrm{~h}$, the reaction mixture was cooled to room temperature and concentrated under reduced pressure. The dark brown residue was dissolved in 1:9 methanol/dichloromethane $(15 \mathrm{~mL})$ and sodium borohydride $(0.700 \mathrm{~g}, 18.5$ 
mmol) was added at room temperature. After $1.5 \mathrm{~h}$, the reaction mixture was poured into water (100 $\mathrm{mL})$ and the layers were separated. The aqueous layer was extracted with dichloromethane $(2 \times 50$ $\mathrm{mL})$. The combined organic extracts were washed with water $(50 \mathrm{~mL})$, dried over anhydrous $\mathrm{Na}_{2} \mathrm{SO} 4$, and concentrated under reduced pressure. The residue was dissolved in dichlormethane $(40 \mathrm{~mL})$, followed by the sequential addition of $\mathrm{NaHCO}_{3}(0.840 \mathrm{~g}, 10.0 \mathrm{mmol})$ and Dess-Martin periodinane $(4.15$ g, $9.20 \mathrm{mmol})$. After $30 \mathrm{~min} .$, a $10 \%$ aqueous solution of $\mathrm{Na}_{2} \mathrm{~S}_{2} \mathrm{O}_{3}(50 \mathrm{~mL})$ was added to the reaction and stirred for $10 \mathrm{~min}$. The resulting mixture was extracted with dichloromethane $(3 \times 50 \mathrm{~mL})$. The organic extracts were combined and washed with water $(100 \mathrm{~mL})$ and brine $(50 \mathrm{~mL})$, dried over anhydrous $\mathrm{Na}_{2} \mathrm{SO}_{4}$, filtered and concentrated under reduced pressure. The residue was purified via flash chromatography $(15 \mathrm{~cm} \times 2.5 \mathrm{~cm} ; 3: 7$ ethyl acetate/hexane $)$ to give 10 as a beige solid $(0.180 \mathrm{~g}, 14 \%$ over 4 steps): $R_{f}=0.42$ (3:7 ethyl acetate/hexane); ${ }^{1} \mathrm{H}$ NMR $\left(400 \mathrm{MHz}, \mathrm{CDCl}_{3}\right) \delta$ 7.70-7.64 (m, 2H), $7.47(\mathrm{t}, J=$ $7.9 \mathrm{~Hz}, 2 \mathrm{H}), 7.39(\mathrm{dd}, J=2.5,1.6 \mathrm{~Hz}, 2 \mathrm{H}), 7.23(\mathrm{ddd}, J=8.2,2.6,1.0 \mathrm{~Hz}, 2 \mathrm{H}), 4.46(\mathrm{~s}, 4 \mathrm{H}), 3.11(\mathrm{~s}, 4 \mathrm{H}) ;{ }^{13} \mathrm{C}$ NMR (101 MHz, $\left.\mathrm{CDCl}_{3}\right) \delta$ 198.82, 157.88, 136.80, 131.14, 123.19, 122.04, 113.29, 64.22, 36.02; HRMS (ESI) calculated for $\mathrm{C}_{18} \mathrm{H}_{17} \mathrm{O}_{4}\left([\mathrm{M}+\mathrm{H}]^{+}\right) m / z=297.1127$, found 297.1113

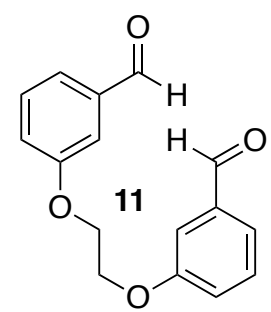

Dialdehyde 11: 3-Hydroxybenzaldehyde (12.2 g, $100 \mathrm{mmol})$ and $\mathrm{KOH}(5.80 \mathrm{~g}, 100$ $\mathrm{mmol})$ were dissolved in ethanol $(125 \mathrm{ml})$ and solvent was removed under vacuum. The residue was dissolved in DMF $(200 \mathrm{ml})$ and 1,2-dibromoethane $(18.5 \mathrm{~g}, 98.6$ mmol) was added slowly. The reaction mixture was heated at $120{ }^{\circ} \mathrm{C}$ for $72 \mathrm{~h}$, at which point water $(400 \mathrm{~mL})$ and $1 \mathrm{M} \mathrm{HCl}(100 \mathrm{~mL})$ were added. The resulting mixture was extracted with ethyl acetate $(3 \times 100 \mathrm{~mL})$. The organic extracts were combined and washed with saturated solution of $\mathrm{NaHCO}_{3}(100 \mathrm{~mL})$ and brine $(100 \mathrm{~mL})$, dried over anhydrous $\mathrm{Na}_{2} \mathrm{SO}_{4}$, filtered and concentrated under reduced pressure. The residue was purified via flash chromatography $(15 \mathrm{~cm} \times$ $5.0 \mathrm{~cm} ; 0.5 \%-2 \%$ acetone/dichloromethane to afford 11 as brown solid. (1.80 g, 13\%): $R_{f}=0.48$ (dichloromethane); ${ }^{1} \mathrm{H}$ NMR (400 MHz, $\left.\mathrm{CDCl}_{3}\right) \delta 10.00$ (s, 2H), 7.53-7.45 (m, 6H), 7.27-7.23 (m, 2H), 4.43 (s, 4H); ${ }^{13} \mathrm{C}$ NMR $\left(151 \mathrm{MHz}, \mathrm{CDCl}_{3}\right) \delta$ 192.20, 159.19, 137.88, 130.33, 124.23, 122.29, 112.73, 66.72. HRMS (ESI) calculated for $\mathrm{C}_{16} \mathrm{H}_{15} \mathrm{O}_{4}\left([\mathrm{M}+\mathrm{H}]^{+}\right) \mathrm{m} / z=271.0970$, found 271.0975 .

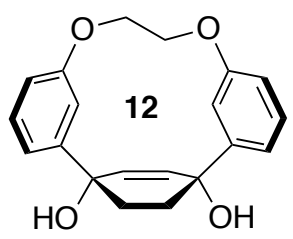

Cyclohex-2-ene-1,4-diol 12: Grubbs second-generation catalyst (0.018 g, $0.020 \mathrm{mmol})$ was added to a stirred solution of syn-allylic diol $17(0.140 \mathrm{~g}, 0.397 \mathrm{mmol})$ in dichloromethane $(12 \mathrm{~mL})$ at $40{ }^{\circ} \mathrm{C}$. After $1.5 \mathrm{~h}$, the reaction was cooled to room 
temperature and the solvent was removed under reduced pressure and residue was purified by flash chromatography $(15 \times 1.3 \mathrm{~cm}, 10-20 \%$ acetone/dichloromethane $)$ to give 12 as an off-white solid (0.102 g, $79 \%) ; R_{f}=0.38\left(1: 4\right.$ acetone/dichloromethane); ${ }^{1} \mathrm{H}$ NMR $\left(600 \mathrm{MHz}, \mathrm{CDCl}_{3}\right) \delta$ 7.40-7.32 (m, 4H), 7.22 (br s, 2H), 6.91-6.84 (m, 2H), $5.88(\mathrm{~s}, 2 \mathrm{H}), 4.52(\mathrm{~d}, J=7.4 \mathrm{~Hz}, 2 \mathrm{H}), 4.17-4.05(\mathrm{~m}, 2 \mathrm{H}), 2.27-2.11(\mathrm{~m}, 4 \mathrm{H})$, 2.11-2.00 (m, 2H); ${ }^{13} \mathrm{C}$ NMR $\left(151 \mathrm{MHz}\right.$, acetone- $\left.d_{6}\right) \delta 157.89,150.28,135.07,130.96,119.77,117.67,112.55$, 73.41, 63.95, 37.02; HRMS (ESI) calculated for $\mathrm{C}_{20} \mathrm{H}_{19} \mathrm{O}_{3}\left(\left[\mathrm{M}-\left(\mathrm{H}_{2} \mathrm{O}\right)+\mathrm{H}\right]^{+}\right) \mathrm{m} / z=307.1334$, found 307.1342

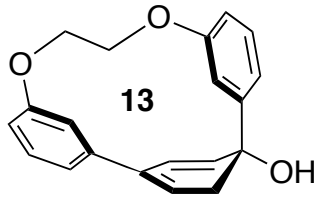

Dienol 13 (monodehydration product): Burgess reagent $(0.016 \mathrm{~g}, 0.066 \mathrm{mmol})$ was added to a stirred solution of $12(0.010 \mathrm{~g}, 0.033 \mathrm{mmol})$ in THF $(2 \mathrm{~mL})$ at $70{ }^{\circ} \mathrm{C}$. After $2.5 \mathrm{~h}$, the reaction was cooled to room temperature, water $(10 \mathrm{~mL})$ was added, and stirred for $5 \mathrm{~min}$. The resulting mixture was extracted with dichloromethane $(3 \times 5 \mathrm{~mL})$. The organic extracts were combined and washed with brine $(5 \mathrm{~mL})$, dried over anhydrous $\mathrm{MgSO}_{4}$, filtered and concentrated under reduced pressure. The residue was purified by flash chromatography ( $5 \times 0.7 \mathrm{~cm}$, dichloromethane to $2 \%$ acetone/dichloromethane) to afford 13 as a colorless solid ( $0.007 \mathrm{~g}$, $68 \%): R_{f}=0.44\left(2 \%\right.$ acetone/dichloromethane); ${ }^{1} \mathrm{H}$ NMR $\left(600 \mathrm{MHz}, \mathrm{CDCl}_{3}\right) \delta$ 7.43-7.35 (m, 2H), 7.30-7.27 $(\mathrm{m}, 1 \mathrm{H}), 7.02-6.95(\mathrm{~m}, 2 \mathrm{H}), 6.95-6.91(\mathrm{~m}, 1 \mathrm{H}), 6.84(\mathrm{dd}, J=8.2,2.8 \mathrm{~Hz}, 1 \mathrm{H}), 6.60-6.54(\mathrm{~m}, 1 \mathrm{H}), 6.29(\mathrm{~d}, J=$ $9.6 \mathrm{~Hz}, 1 \mathrm{H}), 5.91(\mathrm{dd}, J=9.6,1.9 \mathrm{~Hz}, 1 \mathrm{H}), 5.66(\mathrm{ddd}, J=7.3,3.0,1.1 \mathrm{~Hz}, 1 \mathrm{H}), 4.54-4.43(\mathrm{~m}, 1 \mathrm{H}), 4.37-4.25$ (m, 3H), $2.89(\mathrm{dd}, J=15.5,3.1 \mathrm{~Hz}, 1 \mathrm{H}), 2.43(\mathrm{ddd}, J=15.5,7.2,2.0 \mathrm{~Hz}, 1 \mathrm{H}), 2.14(\mathrm{~s}, 1 \mathrm{H})$; ${ }^{13} \mathrm{C}$ NMR (151 $\left.\mathrm{MHz}, \mathrm{CDCl}_{3}\right) \delta 157.11,156.30,143.96,142.12,142.07,134.03,131.13,130.41,130.32,121.60,120.01,118.96$, 117.73, 116.78, 116.44, 116.15, 76.58, 65.43, 64.45, 40.29; HRMS (ESI) calculated for $\mathrm{C}_{20} \mathrm{H}_{19} \mathrm{O}_{3}\left([\mathrm{M}+\mathrm{H}]^{+}\right) \mathrm{m} / z$ $=307.1334$, found 307.1342 .

Alternative procedure: Tin(II) chloride dehydrate $(0.625 \mathrm{~g}, 2.77 \mathrm{mmol})$ was added to a stirred solution of $12(0.040 \mathrm{~g}, 0.14 \mathrm{mmol})$ in $1: 1 \mathrm{THF} / \mathrm{PhMe}(6 \mathrm{~mL})$ at $80{ }^{\circ} \mathrm{C}$. After $24 \mathrm{~h}$, the reaction was cooled to room temperature and $3 \mathrm{M} \mathrm{NaOH}(10 \mathrm{~mL})$ was added, followed by dichloromethane $(10 \mathrm{~mL})$. The layers were separated and the aqueous phase was extracted with dichloromethane $(2 \times 10 \mathrm{~mL})$. The combined organic extracts were washed with brine $(20 \mathrm{~mL})$, dried over anhydrous $\mathrm{Na}_{2} \mathrm{SO}_{4}$, filtered and concentrated under reduced pressure. The residue was purified by flash chromatography $(12 \times 1.3 \mathrm{~cm}$, $4 \%$ acetone/dichloromethane) to give $\mathbf{1 3}$ as colorless solid ( $0.025 \mathrm{~g}, 60 \%)$. 


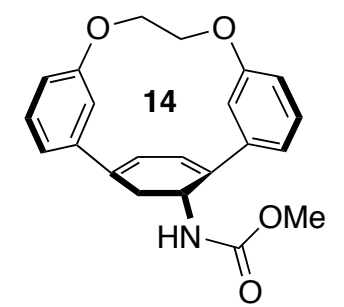

Carbamate 14 (allylic alkylation product): Burgess reagent $(0.055 \mathrm{~g}, 0.23 \mathrm{mmol})$ was added to a stirred solution of $12(0.019 \mathrm{~g}, 0.058 \mathrm{mmol})$ in THF $(2.5 \mathrm{~mL})$ at $50{ }^{\circ} \mathrm{C}$. After $1 \mathrm{~h}$, the reaction was cooled to room temperature, water $(20 \mathrm{~mL})$ was added, and stirred for $5 \mathrm{~min}$. The resulting mixture was extracted with dichloromethane $(3 \times 10 \mathrm{~mL})$. The organic extracts were combined and washed with brine $(10 \mathrm{~mL})$, dried over anhydrous $\mathrm{MgSO}_{4}$, filtered and concentrated under reduced pressure. The residue was purified by flash chromatography $(10 \times 1.0 \mathrm{~cm}$, dichloromethane to $10 \%$ acetone/dichloromethane $)$ to afford $\mathbf{1 4}$ as a white solid (0.008 g, 37\%): $R_{f}=0.39\left(10 \%\right.$ acetone/dichloromethane); ${ }^{1} \mathrm{H}$ NMR $\left(600 \mathrm{MHz}, \mathrm{CDCl}_{3}\right) \delta 7.49$ $7.45(\mathrm{~m}, 1 \mathrm{H})$, 7.43-7.37 (m, 1H), 7.36-7.32 (m, 1H), 7.30-7.24 (m, 1H), 7.03-6.98 (m, 2H), $6.92(\mathrm{~d}, J=7.7 \mathrm{~Hz}$, $1 \mathrm{H}), 6.84(\mathrm{~d}, J=8.4,2.7 \mathrm{~Hz}, 1 \mathrm{H}), 6.67(\mathrm{dd}, J=9.8,2.0 \mathrm{~Hz}, 1 \mathrm{H}), 6.36(\mathrm{dd}, J=9.9,1.3 \mathrm{~Hz}, 1 \mathrm{H}), 6.27(\mathrm{dd}, J=$ 2.7, $1.3 \mathrm{~Hz}, 1 \mathrm{H}), 5.59(\mathrm{ddd}, J=7.3,3.0,1.3 \mathrm{~Hz}, 1 \mathrm{H}), 4.52-4.35(\mathrm{~m}, 2 \mathrm{H}), 4.33-4.19(\mathrm{~m}, 2 \mathrm{H}), 3.85(\mathrm{~s}, 3 \mathrm{H}), 3.38$ $(\mathrm{dd}, J=15.2,3.0 \mathrm{~Hz}, 1 \mathrm{H}), 2.68(\mathrm{ddd}, J=15.2,7.3,2.1 \mathrm{~Hz}, 1 \mathrm{H}) ;{ }^{13} \mathrm{C} \mathrm{NMR}\left(151 \mathrm{MHz}, \mathrm{CDCl}_{3}\right) \delta 157.36$, 156.49, 150.39, 142.43, 141.13, 138.33, 132.42, 130.37, 130.18, 127.79, 121.36, 120.20, 118.86, 118.71, 117.42, 116.83, 116.49, 96.03, 66.97, 64.36, 54.21, 38.48; HRMS (ESI) calculated for $\mathrm{C}_{22} \mathrm{H}_{22} \mathrm{NO}_{4}\left([\mathrm{M}+\mathrm{H}]^{+}\right) \mathrm{m} / \mathrm{z}=$ 364.1549 , found 364.1560

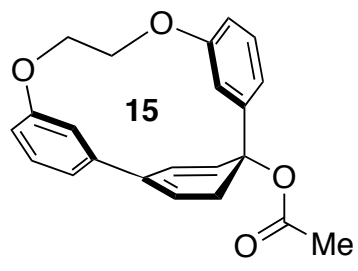

Acetate 15: Acetic anhydride $(0.060 \mathrm{~g}, 0.59 \mathrm{mmol})$ and DMAP $(0.0036 \mathrm{~g}, 0.010$ $\mathrm{mmol})$ were added to a stirred solution of $\mathbf{1 3}(0.030 \mathrm{~g}, 0.098 \mathrm{mmol})$ in pyridine $(2.5 \mathrm{~mL})$ at $40{ }^{\circ} \mathrm{C}$. After $12 \mathrm{~h}$, the reaction was cooled to room temperature and water $(10 \mathrm{~mL})$ was added. The resulting mixture was extracted with dichloromethane $(3 \times 10 \mathrm{~mL})$ and the combined organic extracts were washed with brine $(20 \mathrm{~mL})$, dried over anhydrous $\mathrm{Na}_{2} \mathrm{SO}_{4}$, filtered and concentrated under reduced pressure. The solid residue was purified by flash chromatography $(10 \times 1.3 \mathrm{~cm}$, dichloromethane) to give 15 as colorless solid $(0.026 \mathrm{~g}$, 74\%); $R_{f}=0.49$ (dichloromethane); ${ }^{1} \mathrm{H}$ NMR $\left(400 \mathrm{MHz}, \mathrm{CDCl}_{3}\right) \delta 7.33(\mathrm{~d}, J=7.9 \mathrm{~Hz}, 1 \mathrm{H}), 7.30-7.25(\mathrm{~m}$, $1 \mathrm{H}), 7.07(\mathrm{ddd}, J=7.8,1.8,0.9 \mathrm{~Hz}, 1 \mathrm{H}), 7.00(\mathrm{dd}, J=2.6,1.8 \mathrm{~Hz}, 1 \mathrm{H}), 6.96-6.92(\mathrm{~m}, 1 \mathrm{H}), 6.90(\mathrm{ddd}, J=8.0$, 2.6, $0.9 \mathrm{~Hz}, 1 \mathrm{H}), 6.83(\mathrm{ddd}, J=8.3,2.8,0.9 \mathrm{~Hz}, 1 \mathrm{H}), 6.48(\mathrm{dd}, J=2.9,1.4 \mathrm{~Hz}, 1 \mathrm{H}), 6.37-6.28(\mathrm{~m}, 2 \mathrm{H}), 5.62$ $(\mathrm{ddd}, J=7.3,3.1,1.1 \mathrm{~Hz}, 1 \mathrm{H}), 4.52-4.42(\mathrm{~m}, 1 \mathrm{H}), 4.35(\mathrm{dt}, J=12.8,5.5 \mathrm{~Hz}, 1 \mathrm{H}), 4.27(\mathrm{td}, J=5.7,2.9 \mathrm{~Hz}$, 2H), $3.11(\mathrm{dd}, J=15.4,3.1 \mathrm{~Hz}, 1 \mathrm{H}), 2.43(\mathrm{ddd}, J=15.4,7.2,1.8 \mathrm{~Hz}, 1 \mathrm{H}), 2.14(\mathrm{~s}, 3 \mathrm{H}) ;{ }^{13} \mathrm{C} \mathrm{NMR}(151 \mathrm{MHz}$, $\left.\mathrm{CDCl}_{3}\right) \delta 170.05,157.34,156.53,142.53,141.88,141.76,131.30,130.25,130.17,129.03,120.69,118.87$, $118.79,117.55,117.42,116.33,116.31,83.66,66.09,64.62,38.15,22.30$; HRMS (ESI) calculated for $\mathrm{C}_{22} \mathrm{H}_{21} \mathrm{O}_{4}$ $\left([\mathrm{M}+\mathrm{H}]^{+}\right) m / z=349.1440$, found 349.1428. 


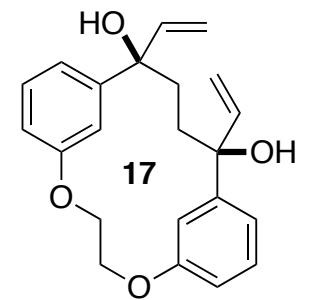
Allylic alcohol 17: Vinylmagnesium chloride (1.6 M in THF, $0.79 \mathrm{~mL}, 1.3 \mathrm{mmol})$ was added to a stirred solution of $10(0.150 \mathrm{~g}, 0.507 \mathrm{mmol})$, in $\mathrm{CH}_{2} \mathrm{Cl}_{2}(10 \mathrm{~mL})$ at $40{ }^{\circ} \mathrm{C}$ in a flame-dried two neck flask. After $30 \mathrm{~min} .$, the reaction mixture was poured directly into water $(40 \mathrm{~mL})$ and further diluted with $1 \mathrm{M} \mathrm{HCl}(20 \mathrm{~mL})$. The resulting mixture was extracted with dichloromethane $(3 \times 15 \mathrm{~mL})$. The organic extracts were combined and washed with a saturated solution of $\mathrm{NaHCO}_{3}(30 \mathrm{~mL})$ and brine $(30 \mathrm{~mL})$, dried over anhydrous $\mathrm{Na}_{2} \mathrm{SO}_{4}$ and concentrated under reduced pressure. The solid residue was purified by flash chromatography (15 $\times 1.3 \mathrm{~cm}, 5-10 \%$ acetone/dichloromethane) to give 17 as a single diastereomer $(0.151 \mathrm{~g}, 75 \%): R_{f}=0.26$ (1:19 acetone/dichloromethane); ${ }^{1} \mathrm{H}$ NMR (400 MHz, $\left.\mathrm{CDCl}_{3}\right) \delta$ 7.33-7.24 (m, 2H), 7.13-7.04 (m, $\left.2 \mathrm{H}\right), 6.94$ $(\mathrm{dd}, J=8.1,2.2 \mathrm{~Hz}, 2 \mathrm{H}), 6.39-6.27(\mathrm{~m}, 2 \mathrm{H}), 6.20(\mathrm{dd}, J=17.2,10.8 \mathrm{~Hz}, 2 \mathrm{H}), 5.30(\mathrm{~d}, J=17.1 \mathrm{~Hz}, 2 \mathrm{H}), 5.15$ $(\mathrm{d}, J=10.7 \mathrm{~Hz}, 2 \mathrm{H}), 4.45-4.27(\mathrm{~m}, 4 \mathrm{H}), 1.88(\mathrm{~s}, 2 \mathrm{H}), 1.84-1.69(\mathrm{~m}, 4 \mathrm{H}) ;{ }^{13} \mathrm{C} \mathrm{NMR}\left(101 \mathrm{MHz}, \mathrm{CDCl}_{3}\right) \delta$ 158.67, 145.60, 143.29, 129.51, 120.01, 116.74, 114.58, 113.24, 77.39, 68.34, 36.78; HRMS (ESI) calculated for $\mathrm{C}_{22} \mathrm{H}_{25} \mathrm{O}_{4}\left(\left[\mathrm{M}-\left(2 \mathrm{H}_{2} \mathrm{O}\right)+\mathrm{H}\right]^{+}\right) \mathrm{m} / z=317.1542$, found 317.1539 . 
3. ${ }^{1} \mathrm{H}$ and ${ }^{13} \mathrm{C}$ NMR Spectra (listed in numerical order)

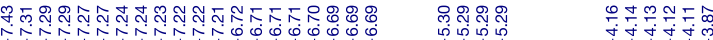
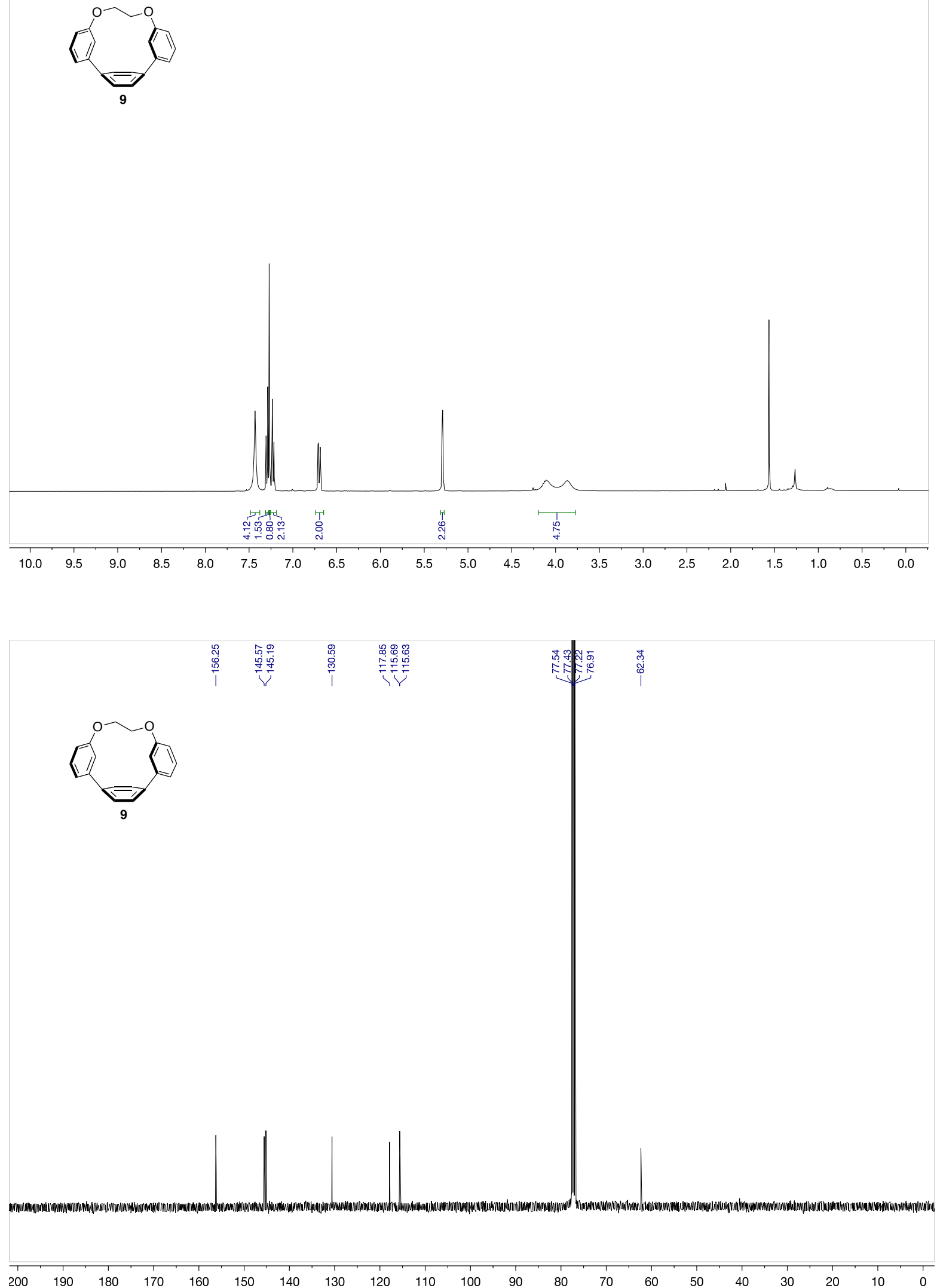

SI-7 


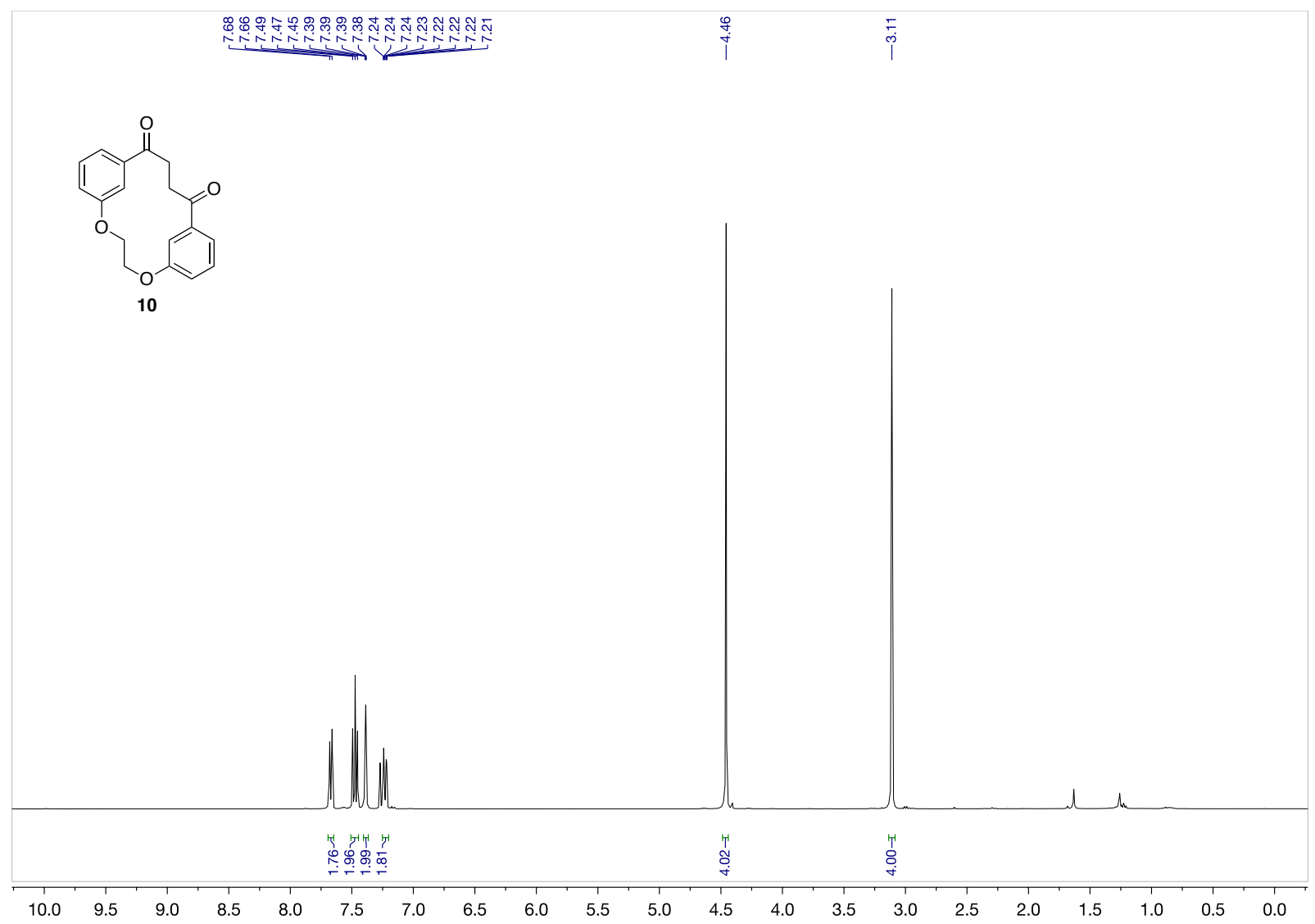

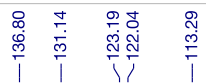

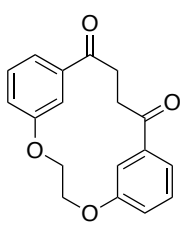

10

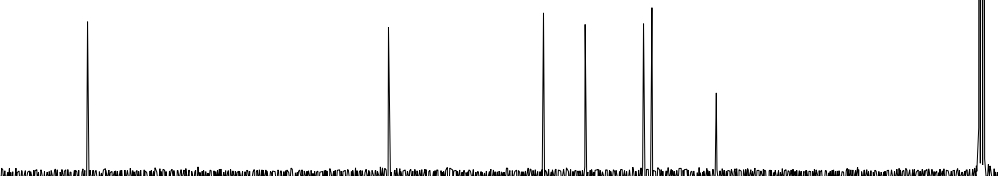

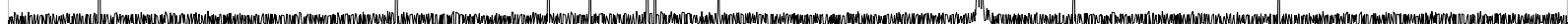

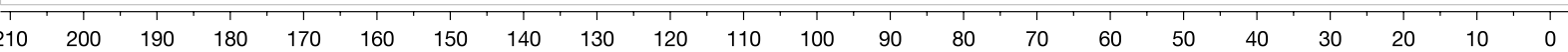

SI-8 

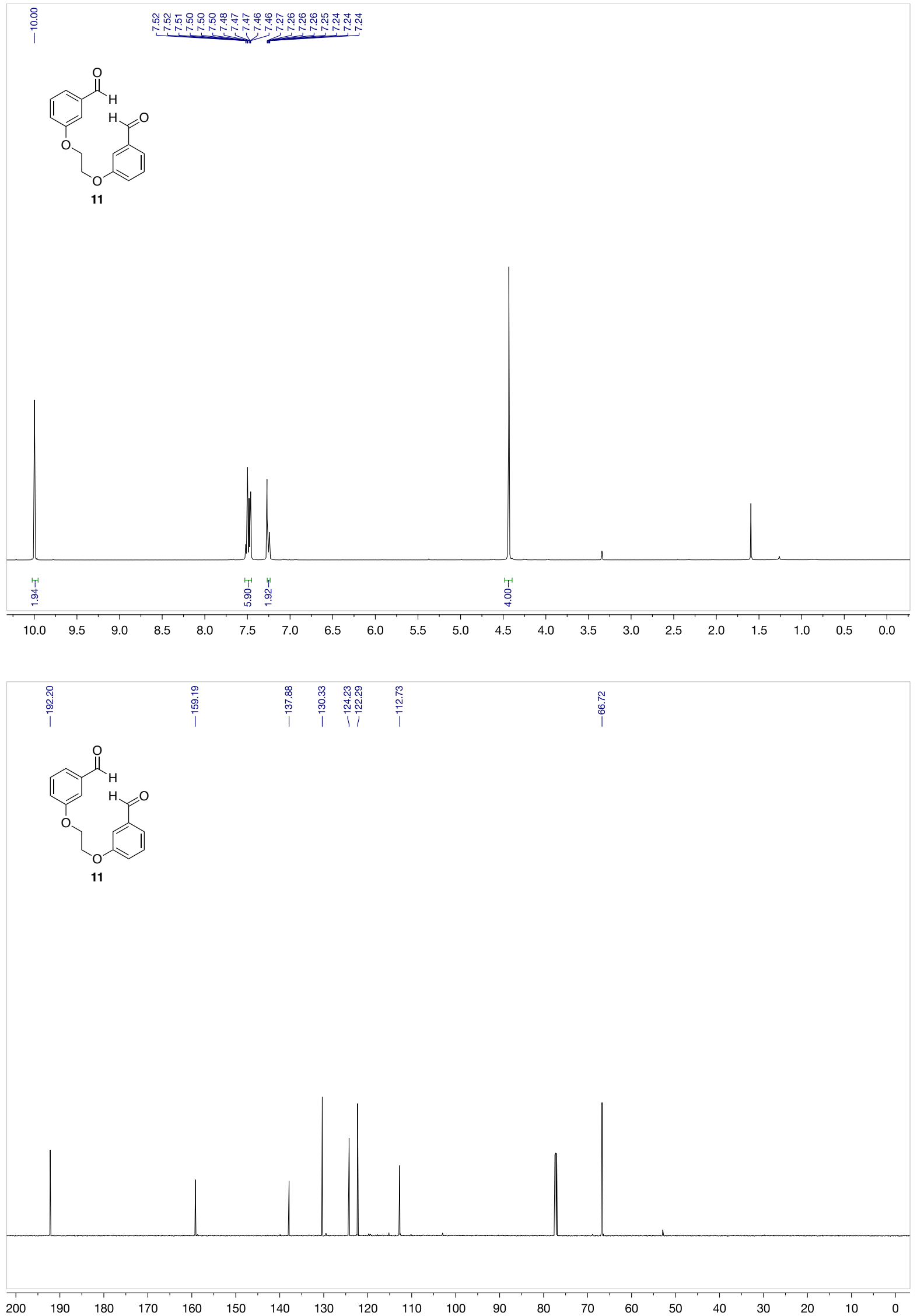

SI-9 

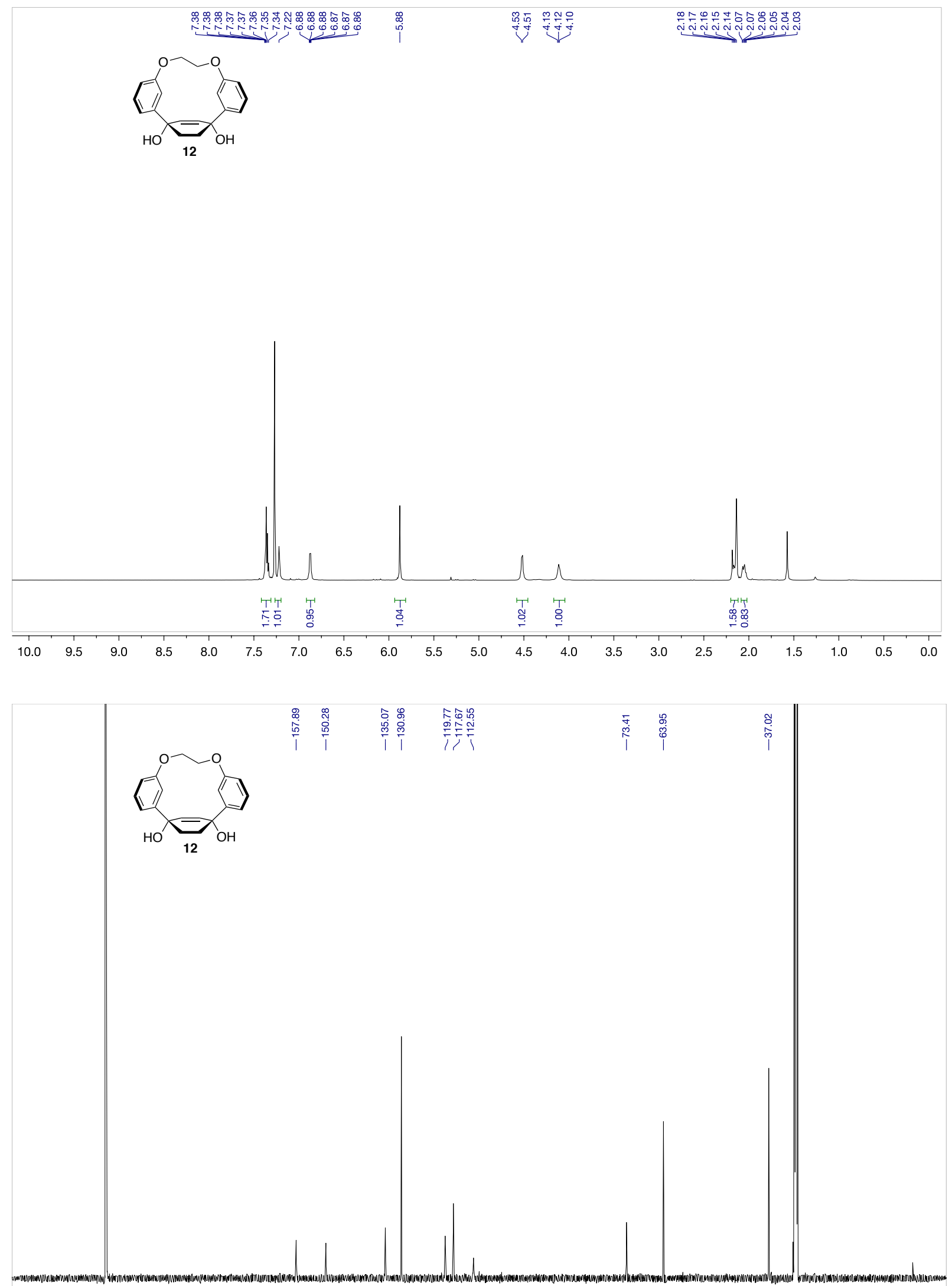

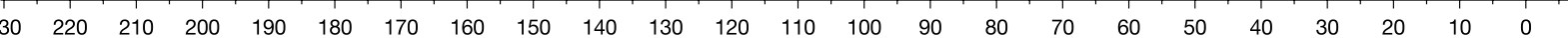

SI-10 

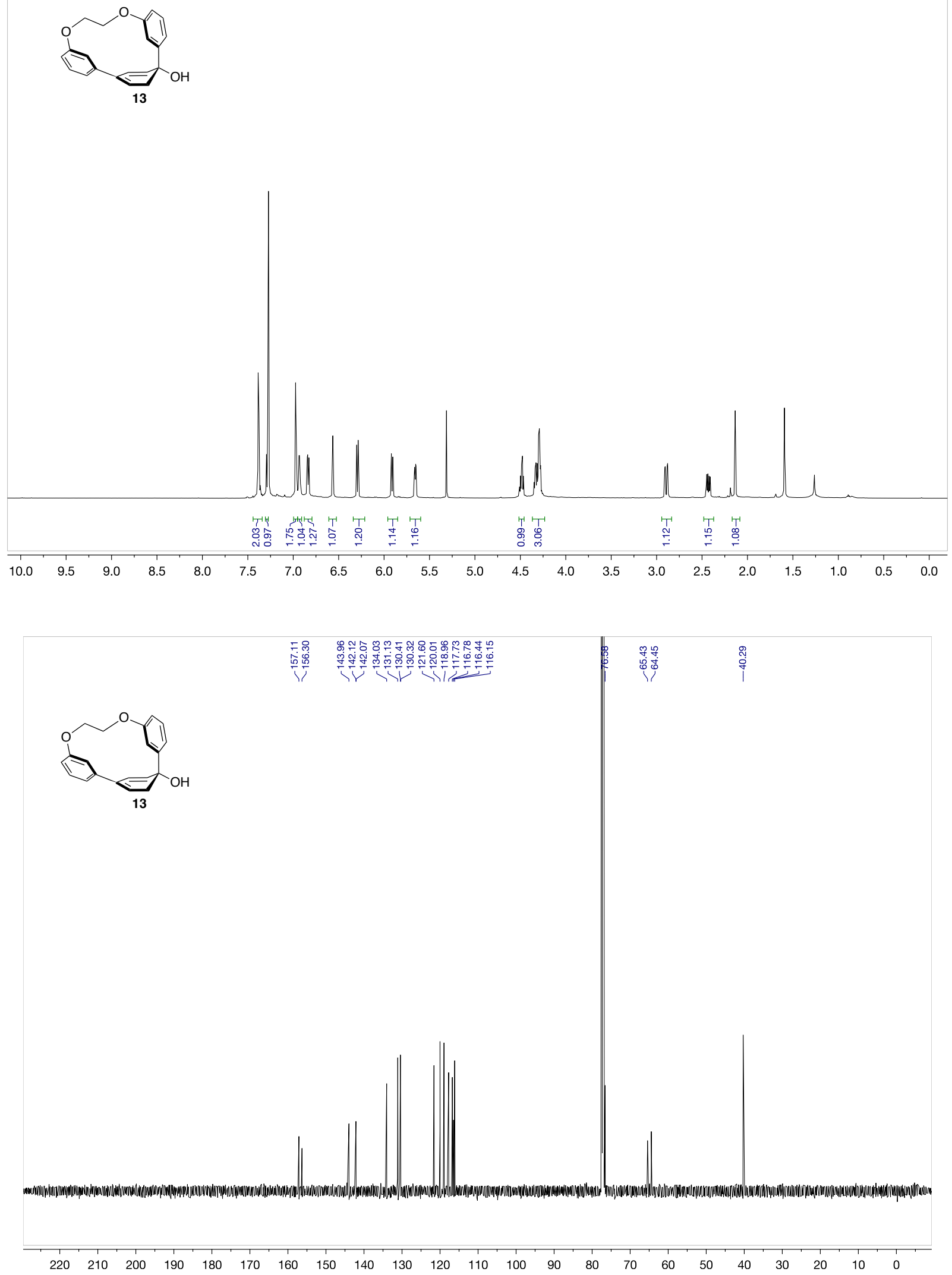

SI-11 

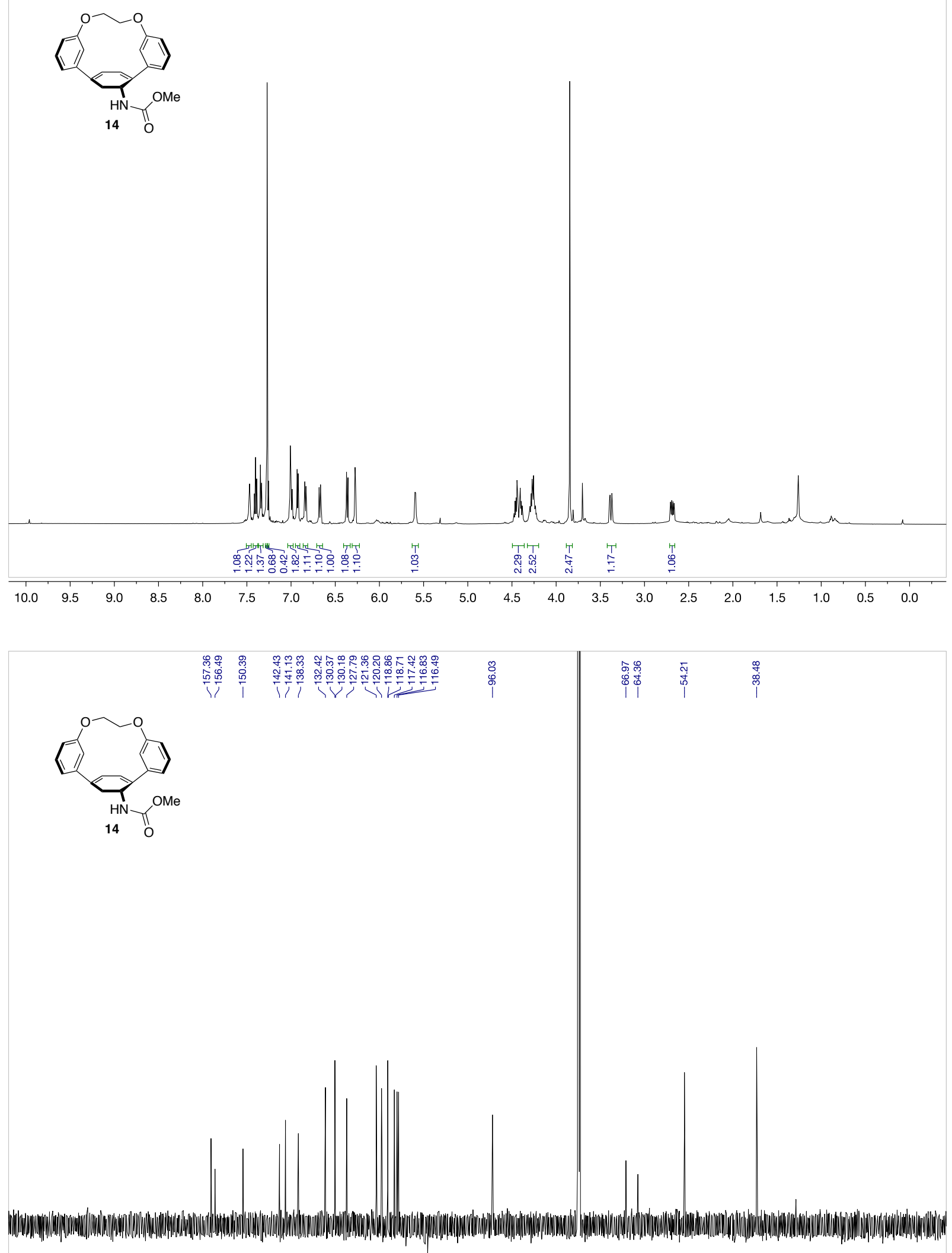

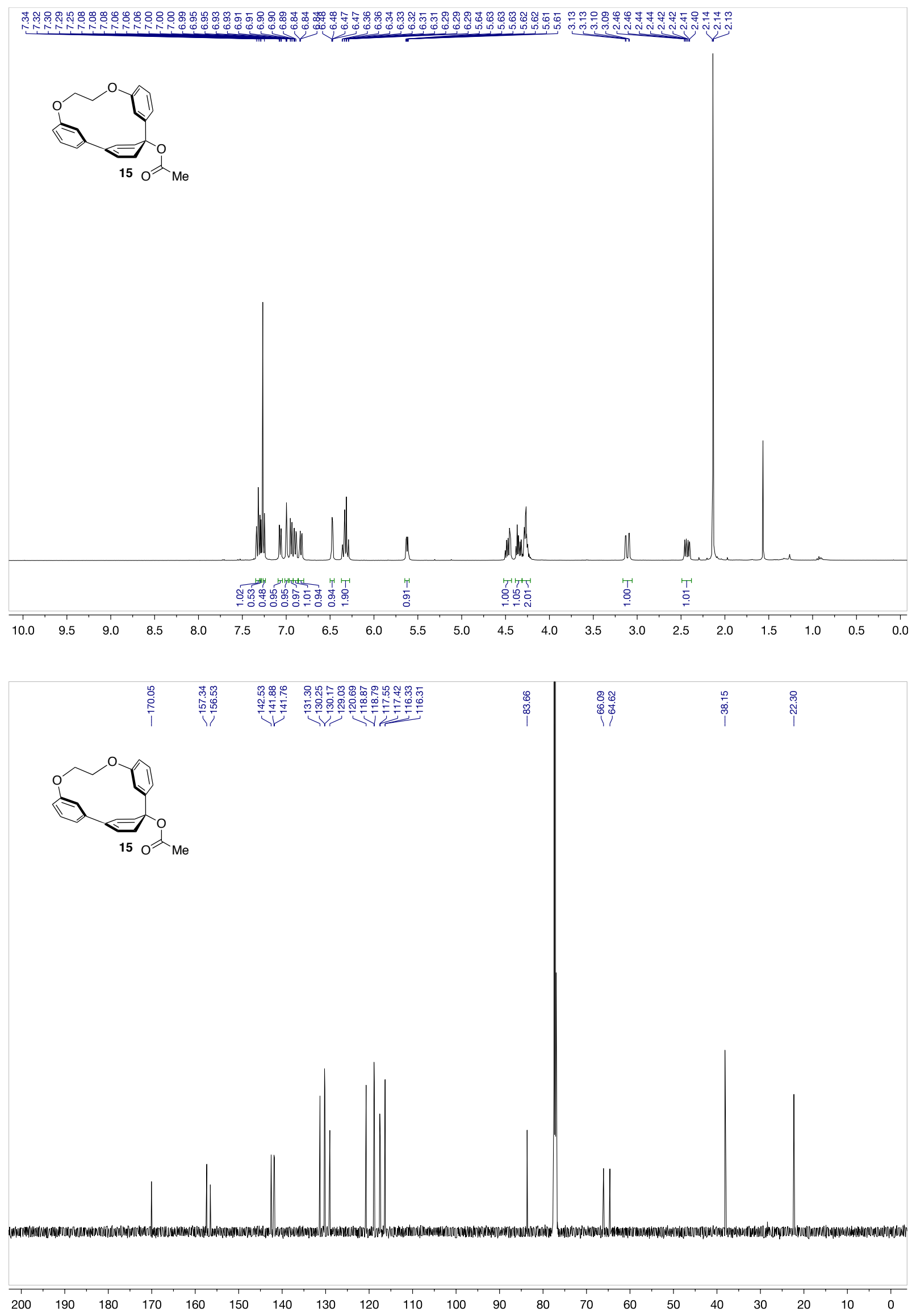

SI-13 

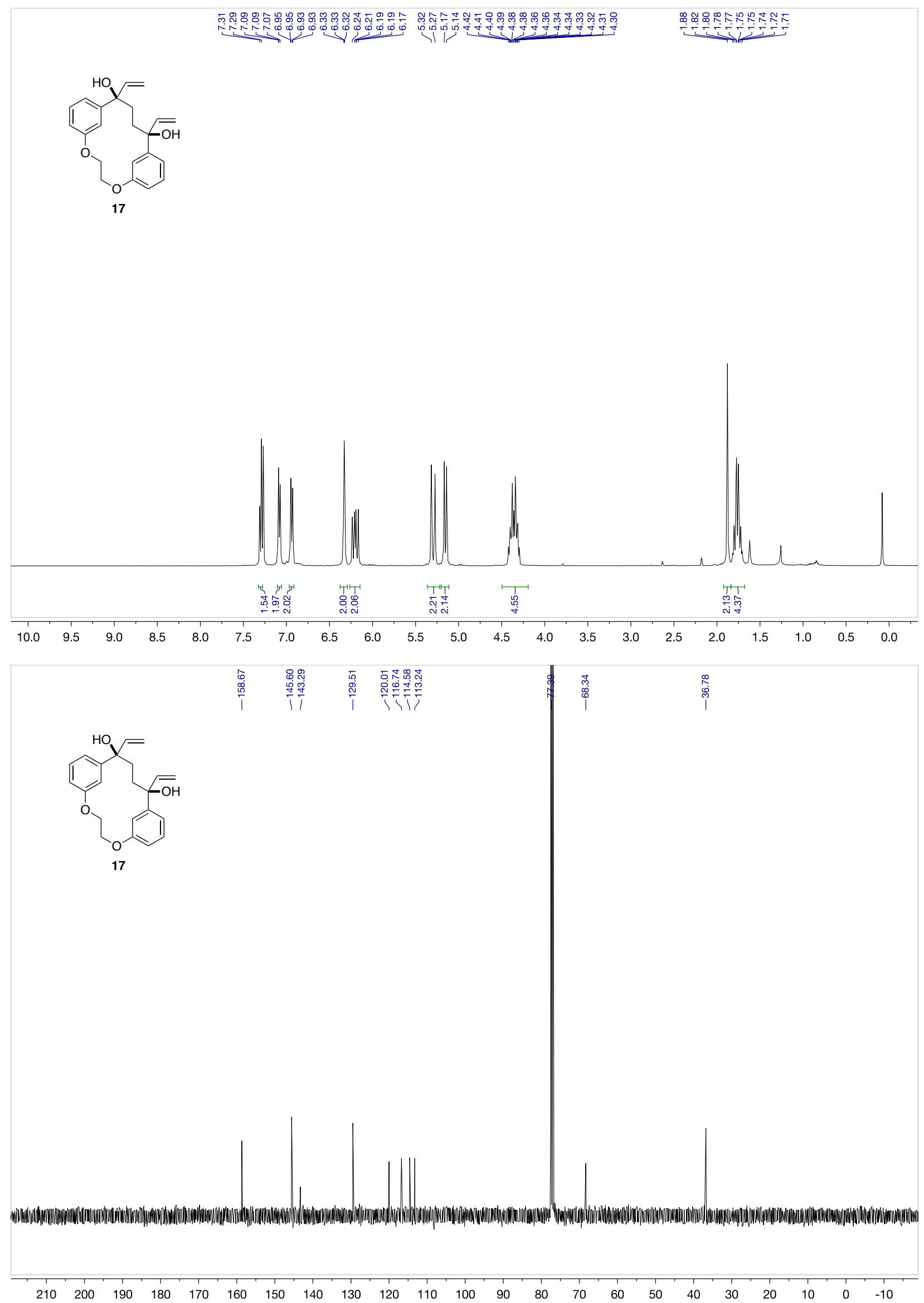

SI-14 


\section{DFT Computed Strain Energies of 31 and 34}

Full geometry optimization was performed using the B3LYP functional in conjunction with a 6-31G(d) basis set. The harmonic vibrational analysis was done at the same level to verify the nature of the stationary point. All of the electronic calculations were performed with Gaussian $09^{1}$ package of programs.

Cartesian Coordinates for 9 (in $\AA$ ):

$\begin{array}{lrrr}\mathrm{O} & 1.3507530 & 2.5894540 & 0.1399780 \\ \mathrm{O} & -1.6065860 & 2.2466540 & -0.9964240 \\ \mathrm{C} & -3.5616810 & 1.1896790 & 0.0587400 \\ \mathrm{H} & -4.0585740 & 2.1425690 & 0.2166250 \\ \mathrm{C} & -2.3083730 & 1.1333920 & -0.5641370 \\ \mathrm{C} & -1.7113940 & -0.1003270 & -0.7842810 \\ \mathrm{H} & -0.7340510 & -0.1021470 & -1.2489490 \\ \mathrm{C} & -2.2573120 & -1.3009430 & -0.2843280 \\ \mathrm{C} & -1.2001430 & -2.3613140 & -0.1666620 \\ \mathrm{C} & -0.6544860 & -2.5184700 & 1.1222820 \\ \mathrm{C} & 0.7265060 & -2.4212640 & 1.3021070 \\ \mathrm{C} & 1.5589460 & -2.1777760 & 0.1950370 \\ \mathrm{C} & 2.4565130 & -0.9672810 & 0.2353300 \\ \mathrm{C} & 3.8098060 & -0.8120170 & -0.0615980 \\ \mathrm{H} & 4.4552660 & -1.6762940 & -0.1876020 \\ \mathrm{C} & 4.3215170 & 0.4858750 & -0.2213310 \\ \mathrm{H} & 5.3770680 & 0.6149410 & -0.4468590 \\ \mathrm{C} & 3.5127950 & 1.6191950 & -0.1257700 \\ \mathrm{H} & 3.9169860 & 2.6131620 & -0.2903370 \\ \mathrm{C} & 2.1475810 & 1.4680320 & 0.1636890 \\ \mathrm{C} & 0.1002740 & 2.6259540 & 0.8423430 \\ \mathrm{C} & -1.0711650 & 3.1346170 & -0.0094200 \\ \mathrm{C} & 1.6569060 & 0.1872080 & 0.3809050 \\ \mathrm{H} & 0.6029630 & 0.0412380 & 0.5620210 \\ \mathrm{C} & 1.0423810 & -2.5047100 & -1.0729350 \\ \mathrm{C} & -0.3383220 & -2.5961150 & -1.2538590 \\ \mathrm{C} & -3.5109020 & -1.2416300 & 0.3358080 \\ \mathrm{H} & -3.9683420 & -2.1418550 & 0.7370920 \\ \mathrm{C} & -4.1573730 & -0.0058220 & 0.4752280 \\ \mathrm{H} & -5.1348850 & 0.0301810 & 0.9493880 \\ \mathrm{H} & -1.3005610 & -2.4309670 & 1.9928070 \\ & -0.7336710 & -2.5807350 & -2.2667920 \\ \mathrm{H} & 1.6791350 & -2.4161220 & -1.9501310 \\ \mathrm{H} & -1.1110800 & -2.2661350 & 2.3074080 \\ \mathrm{H} & -0.1451910 & 1.6611770 & 1.2907710 \\ \mathrm{H} & & 3.4414140 & 0.6915210\end{array}$


$\mathrm{H} \quad-0.7523670 \quad 4.0164370 \quad-0.5719020$

Cartesian Coordinates for $p$-terphenyl system of 9 (in $\AA$ ):

$\begin{array}{lrrr}\mathrm{C} & 5.7298080 & -0.0000250 & -0.0000490 \\ \mathrm{H} & 6.8164560 & -0.0000720 & -0.0000650 \\ \mathrm{C} & 5.0258830 & 0.9516320 & -0.7402510 \\ \mathrm{C} & 3.6318610 & 0.9511110 & -0.7403620 \\ \mathrm{H} & 3.0948170 & 1.6791580 & -1.3419920 \\ \mathrm{C} & 2.9088170 & 0.0000650 & 0.0000190 \\ \mathrm{C} & 1.4244360 & 0.0000400 & 0.0000370 \\ \mathrm{C} & 0.6957260 & -1.2010750 & -0.0000990 \\ \mathrm{C} & -0.6956650 & -1.2011040 & 0.0000450 \\ \mathrm{C} & -1.4244540 & -0.0000280 & 0.0000380 \\ \mathrm{C} & -2.9088030 & -0.0000600 & 0.0000190 \\ \mathrm{C} & -3.6318470 & 0.9513120 & 0.7400090 \\ \mathrm{H} & -3.0947600 & 1.6795440 & 1.3413850 \\ \mathrm{C} & -5.0258520 & 0.9518960 & 0.7398580 \\ \mathrm{H} & -5.5631350 & 1.6925710 & 1.3265430 \\ \mathrm{C} & -5.7298080 & 0.0000040 & -0.0000450 \\ \mathrm{H} & -6.8164570 & 0.0000370 & -0.0000420 \\ \mathrm{C} & -5.0258720 & -0.9518830 & -0.7399420 \\ \mathrm{C} & -3.6318530 & -0.9513420 & -0.7400640 \\ \mathrm{H} & -3.0948130 & -1.6795620 & -1.3414890 \\ \mathrm{C} & -0.6957340 & 1.2010810 & 0.0000900 \\ \mathrm{C} & 0.6956540 & 1.2011210 & 0.0002320 \\ \mathrm{C} & 3.6318460 & -0.9510750 & 0.7403170 \\ \mathrm{H} & 3.0947660 & -1.6791190 & 1.3419210 \\ \mathrm{C} & 5.0258550 & -0.9516650 & 0.7401690 \\ \mathrm{H} & 5.5631100 & -1.6921350 & 1.3271390 \\ \mathrm{H} & 1.2271680 & -2.1485950 & -0.0179600 \\ \mathrm{H} & 1.2270420 & 2.1486690 & 0.0181750 \\ \mathrm{H} & -1.2271660 & 2.1486150 & -0.0176010 \\ \mathrm{H} & -1.2270310 & -2.1486690 & 0.0178270 \\ & 5.5631360 & 1.6920560 & -1.3272850 \\ -5.5631340 & -1.6925250 & -1.3266920\end{array}$

Cartesian Coordinates for alkoxy bridging group of 9 (in $\AA$ ):

$\begin{array}{lrrr}\mathrm{O} & 1.4176710 & -0.5825310 & -0.1626370 \\ \mathrm{O} & -1.3723170 & -0.5991420 & 0.0514650 \\ \mathrm{C} & 0.7363910 & 0.5764150 & 0.2772840 \\ \mathrm{C} & -0.6824450 & 0.6105770 & -0.2811220 \\ \mathrm{H} & 1.3070760 & 1.4472320 & -0.0653310 \\ \mathrm{H} & 0.6954220 & 0.6234740 & 1.3822480 \\ \mathrm{H} & -1.2290720 & 1.4986120 & 0.0700240\end{array}$




$\begin{array}{lrrr}\mathrm{H} & -0.6502730 & 0.6336770 & -1.3743790 \\ \mathrm{H} & -1.5707600 & -0.5727180 & 1.0013810 \\ \mathrm{H} & 0.7610990 & -1.2988370 & -0.1015400\end{array}$

Cartesian Coordinates for 12 (in $\AA$ ):

$\begin{array}{lrrr}\mathrm{C} & -1.2791850 & 2.9559350 & 0.8969770 \\ \mathrm{H} & -0.9497100 & 2.2320100 & 1.6511080 \\ \mathrm{H} & -1.4301710 & 3.9138230 & 1.4013550 \\ \mathrm{C} & -0.2268460 & 3.0990440 & -0.2168670 \\ \mathrm{H} & -0.2903710 & 2.2533330 & -0.9077120 \\ \mathrm{H} & -0.4167310 & 4.0152940 & -0.7864240 \\ \mathrm{C} & 3.1241010 & 2.3874870 & -0.6726180 \\ \mathrm{H} & 3.3579910 & 3.4004810 & -0.9846480 \\ \mathrm{C} & 3.9603510 & 1.3131720 & -0.9883040 \\ \mathrm{H} & 4.8760070 & 1.4961560 & -1.5446060 \\ \mathrm{C} & 3.6169250 & 0.0066220 & -0.6369760 \\ \mathrm{H} & 4.2538610 & -0.8245370 & -0.9231660 \\ \mathrm{C} & 0.9957530 & -1.8001480 & 1.5058480 \\ \mathrm{H} & 1.4008640 & -1.7544810 & 2.5171820 \\ \mathrm{C} & -0.3185810 & -1.9749380 & 1.3281430 \\ \mathrm{H} & -0.9763850 & -2.0901080 & 2.1878010 \\ \mathrm{C} & -3.4403450 & -1.3872590 & -0.5464310 \\ \mathrm{H} & -3.6869150 & -2.4142010 & -0.7880290 \\ \mathrm{C} & -4.4003410 & -0.3715780 & -0.6609150 \\ \mathrm{H} & -5.4038860 & -0.6234630 & -0.9942880 \\ \mathrm{C} & -4.1024640 & 0.9551230 & -0.3597530 \\ \mathrm{H} & -4.8468930 & 1.7399190 & -0.4504530 \\ \mathrm{C} & -1.8614410 & 0.2710130 & 0.1980490 \\ \mathrm{H} & -0.8640270 & 0.5045690 & 0.5231240 \\ \mathrm{C} & 0.0025930 & -1.8361530 & -1.1794180 \\ \mathrm{H} & -0.4067800 & -2.3135980 & -2.0759750 \\ \mathrm{H} & 0.0643570 & -0.7642100 & -1.3931700 \\ \mathrm{C} & 1.4107310 & -2.3593020 & -0.8750030 \\ \mathrm{H} & 2.0733630 & -2.1948920 & -1.7310290 \\ \mathrm{H} & 1.3994170 & -3.4399630 & -0.6819040 \\ \mathrm{C} & 1.6548890 & 0.8471590 & 0.4704600 \\ \mathrm{C} & 0.8007670 & 0.6895890 & 1.1134470 \\ \mathrm{C} & 1.9521880 & 2.1458440 & 0.0446540 \\ \mathrm{C} & 2.4424200 & -0.2425890 & 0.0891100 \\ \mathrm{C} & 2.0123780 & -1.6926920 & 0.3818340 \\ \mathrm{C} & -1.0098080 & -2.0749230 & -0.0229990 \\ \mathrm{H} & 3.6547700 & -2.0239340 & 1.4020670\end{array}$




$\begin{array}{lrrr}\mathrm{H} & -0.8869840 & -4.0253120 & 0.0088500 \\ \mathrm{O} & -2.5595180 & 2.6017830 & 0.3691240 \\ \mathrm{O} & 1.1043270 & 3.2010930 & 0.3229140 \\ \mathrm{O} & 3.1645010 & -2.4902410 & 0.7049290 \\ \mathrm{O} & -1.6001750 & -3.3816340 & -0.1264610\end{array}$

Cartesian Coordinates for $p$-terphenyl precursor of 12 (in $\AA$ ):

$\begin{array}{lrrr}\mathrm{C} & 4.0940560 & 2.3202700 & -0.2897090 \\ \mathrm{H} & 4.7116610 & 3.2017250 & -0.4398880 \\ \mathrm{C} & 4.5108690 & 1.0778610 & -0.7740660 \\ \mathrm{H} & 5.4561020 & 0.9886220 & -1.3034310 \\ \mathrm{C} & 3.7190490 & -0.0525680 & -0.5794970 \\ \mathrm{H} & 4.0468640 & -1.0193360 & -0.9498210 \\ \mathrm{C} & 0.6684060 & -1.1279900 & 1.4258850 \\ \mathrm{H} & 1.1190800 & -1.1546090 & 2.4186790 \\ \mathrm{C} & -0.6603550 & -1.0388480 & 1.3072890 \\ \mathrm{H} & -1.2900770 & -1.0228780 & 2.1948120 \\ \mathrm{C} & -3.8375290 & -0.3927820 & -0.3702590 \\ \mathrm{H} & -4.0492520 & -1.4293710 & -0.6033250 \\ \mathrm{C} & -4.8585900 & 0.5599910 & -0.3971650 \\ \mathrm{H} & -5.8686060 & 0.2546060 & -0.6589070 \\ \mathrm{C} & -4.5904500 & 1.8935140 & -0.0887690 \\ \mathrm{H} & -5.3871310 & 2.6325390 & -0.1085050 \\ \mathrm{C} & -2.2696490 & 1.3202690 & 0.2731420 \\ \mathrm{H} & -1.2605020 & 1.6259620 & 0.5382760 \\ \mathrm{C} & -0.4043270 & -0.7748720 & -1.1811720 \\ \mathrm{H} & -0.9098300 & -1.0205460 & -2.1209340 \\ \mathrm{H} & -0.1624290 & 0.2937550 & -1.2140900 \\ \mathrm{C} & 0.8847380 & -1.5864890 & -1.0293120 \\ \mathrm{H} & 1.5429220 & -1.4387960 & -1.8914550 \\ \mathrm{H} & 0.6589390 & -2.6597970 & -0.9869990 \\ \mathrm{C} & 2.0888460 & 1.2851440 & 0.5863360 \\ \mathrm{H} & 1.1538060 & 1.3711580 & 1.1316200 \\ \mathrm{C} & 2.8813910 & 2.4193000 & 0.3912450 \\ \mathrm{C} & 2.4938670 & 0.0361170 & 0.1005260 \\ \mathrm{C} & 1.6435940 & -1.2335400 & 0.2670500 \\ \mathrm{C} & -1.3942970 & -1.0446820 & -0.0227280 \\ \mathrm{C} & -2.5302650 & -0.0221490 & -0.0363150 \\ \mathrm{C} & -3.2889780 & 2.2711150 & 0.2480030 \\ \mathrm{H} & -1.1182600 & -2.1208600 & 1.2152060 \\ \mathrm{H} & 2.5088600 & -2.3643970 & 0.4985770 \\ \mathrm{H} & -2.0024200 & -2.3414450 & -0.1841480 \\ \mathrm{H} & & 3.3793620 & 0.7779840 \\ \mathrm{H} & -3.3063770 & 0.4936110\end{array}$


Cartesian Coordinates for alkoxy bridging group of $\mathbf{1 2}$ (in $\AA$ ):

$\begin{array}{lrrr}\mathrm{C} & 0.5720490 & 0.5086490 & 0.0286860 \\ \mathrm{H} & 0.4719770 & 1.1824520 & -0.8414100 \\ \mathrm{H} & 0.5065830 & 1.1277890 & 0.9279790 \\ \mathrm{C} & -0.5720500 & -0.5086490 & 0.0286980 \\ \mathrm{H} & -0.4719380 & -1.1826730 & -0.8412340 \\ \mathrm{H} & -0.5065560 & -1.1275960 & 0.9281100 \\ \mathrm{O} & 1.8484100 & -0.1139930 & 0.0632340 \\ \mathrm{O} & -1.8484070 & 0.1140020 & 0.0632250 \\ \mathrm{H} & 1.9608130 & -0.6066390 & -0.7647110 \\ \mathrm{H} & -1.9608930 & 0.6066010 & -0.7647100\end{array}$

Cartesian Coordinates for $\mathbf{1 3}$ (in $\AA$ ):

$\begin{array}{lrrr}\mathrm{C} & -2.1188000 & 2.3709140 & 0.9891240 \\ \mathrm{H} & -1.5709690 & 1.9082010 & 1.8179640 \\ \mathrm{H} & -2.4665200 & 3.3471390 & 1.3342790 \\ \mathrm{C} & -1.2026870 & 2.5224570 & -0.2417570 \\ \mathrm{H} & -1.1015980 & 1.5653670 & -0.7591890 \\ \mathrm{H} & -1.6455810 & 3.2417980 & -0.9401610 \\ \mathrm{C} & 2.3181910 & 2.8436180 & -0.6795750 \\ \mathrm{H} & 2.2790830 & 3.8988810 & -0.9307600 \\ \mathrm{C} & 3.4580820 & 2.0767000 & -0.9187280 \\ \mathrm{H} & 4.3309400 & 2.5452230 & -1.3662100 \\ \mathrm{C} & 3.4905110 & 0.7139810 & -0.6091650 \\ \mathrm{H} & 4.3774280 & 0.1258590 & -0.8209310 \\ \mathrm{C} & 1.5740470 & -1.7416480 & 1.5440590 \\ \mathrm{H} & 2.0668390 & -1.6913000 & 2.5131170 \\ \mathrm{C} & 0.2769830 & -2.0619460 & 1.4333650 \\ \mathrm{H} & -0.3233240 & -2.2739700 & 2.3157930 \\ \mathrm{C} & -2.7560250 & -2.1872350 & -0.8715270 \\ \mathrm{H} & -2.5706290 & -3.1339260 & -1.3704980 \\ \mathrm{C} & -3.9433700 & -1.4823920 & -1.1048200 \\ \mathrm{H} & -4.6981290 & -1.9092030 & -1.7602300 \\ \mathrm{C} & -4.1525090 & -0.2114640 & -0.5679320 \\ \mathrm{H} & -5.0314470 & 0.3734670 & -0.8200760 \\ \mathrm{C} & -2.0753200 & -0.4309750 & 0.6318170 \\ \mathrm{H} & -1.3479770 & -0.0340850 & 1.3257700 \\ \mathrm{C} & 0.3995020 & -2.2413450 & -0.9799990 \\ \mathrm{H} & -0.0382260 & -2.2223200 & -1.9745720 \\ \mathrm{C} & 1.9027990 & -2.2896370 & -0.8528600 \\ \mathrm{H} & 2.3801600 & -1.9685580 & -1.7839590 \\ \mathrm{H} & 2.2515370 & -3.3143450 & -0.6501500 \\ \mathrm{C} & 1.2420910 & 0.8808200 & 0.2332100\end{array}$




$\begin{array}{lrrr}\mathrm{H} & 0.3897180 & 0.4347310 & 0.7180610 \\ \mathrm{C} & 1.1956820 & 2.2343950 & -0.1116610 \\ \mathrm{C} & 2.3768700 & 0.0997940 & -0.0204560 \\ \mathrm{C} & 2.4139510 & -1.4115270 & 0.3169970 \\ \mathrm{C} & -0.3821590 & -2.0990110 & 0.1097100 \\ \mathrm{C} & -1.7956700 & -1.6476670 & -0.0091950 \\ \mathrm{C} & -3.1790840 & 0.3373580 & 0.2766800 \\ \mathrm{H} & 4.1660450 & -1.2346250 & 1.1700150 \\ \mathrm{O} & -3.3199970 & 1.6478270 & 0.6733820 \\ \mathrm{O} & 0.0888810 & 3.0254060 & 0.1288770 \\ \mathrm{O} & 3.7678840 & -1.8369160 & 0.5199920\end{array}$

Cartesian Coordinates for $p$-terphenyl precursor of 13 (in $\AA$ ):

$\begin{array}{lrrr}\mathrm{C} & 3.9626990 & 2.3160910 & -0.4566860 \\ \mathrm{H} & 4.5472680 & 3.2094480 & -0.6601790 \\ \mathrm{C} & 4.4683200 & 1.0553810 & -0.7793170 \\ \mathrm{H} & 5.4501590 & 0.9618440 & -1.2366460 \\ \mathrm{C} & 3.7176080 & -0.0901820 & -0.5152440 \\ \mathrm{H} & 4.1102750 & -1.0700230 & -0.7691160 \\ \mathrm{C} & 0.7001370 & -1.1499520 & 1.5079440 \\ \mathrm{H} & 1.1434320 & -1.2772700 & 2.4941080 \\ \mathrm{C} & -0.6044240 & -0.8722590 & 1.3724430 \\ \mathrm{H} & -1.2371830 & -0.7896750 & 2.2529250 \\ \mathrm{C} & -3.5089940 & -0.5369840 & -1.0190440 \\ \mathrm{H} & -3.2178070 & -1.3250130 & -1.7077280 \\ \mathrm{C} & -4.7836320 & 0.0189750 & -1.1057900 \\ \mathrm{H} & -5.4698540 & -0.3274610 & -1.8743340 \\ \mathrm{C} & -5.1856720 & 1.0040450 & -0.2010220 \\ \mathrm{H} & -6.1817310 & 1.4336550 & -0.2653040 \\ \mathrm{C} & -3.0237180 & 0.8690210 & 0.8765660 \\ \mathrm{H} & -2.3389640 & 1.2193090 & 1.6442730 \\ \mathrm{C} & -0.5260470 & -1.0499190 & -1.0424390 \\ \mathrm{H} & -0.9466710 & -0.9244010 & -2.0367470 \\ \mathrm{C} & 0.8326150 & -1.6896880 & -0.9372580 \\ \mathrm{H} & 1.4316120 & -1.4892040 & -1.8312560 \\ \mathrm{H} & 0.7080620 & -2.7836370 & -0.8848790 \\ \mathrm{C} & 1.9506720 & 1.2722690 & 0.3957010 \\ \mathrm{H} & 0.9731220 & 1.3641460 & 0.8571970 \\ \mathrm{C} & 2.7007240 & 2.4193740 & 0.1291230 \\ \mathrm{C} & 2.4498910 & 0.0028420 & 0.0791300 \\ \mathrm{C} & 1.6371830 & -1.2822170 & 0.3202040 \\ \mathrm{C} & -1.2325770 & -0.6873200 & 0.0487700 \\ \mathrm{C} & -2.6002270 & -0.1161570 & -0.0322720 \\ \mathrm{C} & -4.2997770 & 1.4252380 & 0.7916140\end{array}$




$\begin{array}{lrrr}\mathrm{H} & 3.1549690 & -2.1234320 & 1.2337840 \\ \mathrm{O} & 2.5310480 & -2.3897050 & 0.5380890 \\ \mathrm{H} & 2.2963590 & 3.3957750 & 0.3837490 \\ \mathrm{H} & -4.6001210 & 2.1922350 & 1.5008970\end{array}$

Cartesian Coordinates for alkoxy bridging group of $\mathbf{1 3}$ (in $\AA$ ):

$\begin{array}{lrrr}\mathrm{C} & 0.5720300 & 0.5086360 & 0.0283440 \\ \mathrm{H} & 0.4723770 & 1.1822250 & -0.8419680 \\ \mathrm{H} & 0.5062160 & 1.1280430 & 0.9274350 \\ \mathrm{C} & -0.5720490 & -0.5086340 & 0.0283160 \\ \mathrm{H} & -0.4723010 & -1.1822340 & -0.8420020 \\ \mathrm{H} & -0.5062030 & -1.1280580 & 0.9273870 \\ \mathrm{O} & 1.8483250 & -0.1140460 & 0.0636410 \\ \mathrm{O} & -1.8483280 & 0.1140080 & 0.0636200 \\ \mathrm{H} & 1.9614880 & -0.6060840 & -0.7645370 \\ \mathrm{H} & -1.9614360 & 0.6064000 & -0.7643600\end{array}$

[1] Gaussian 09, Revision D.01: M. J. Frisch, G. W. Trucks, H. B. Schlegel, G. E. Scuseria, M. A. Robb, J. R. Cheeseman, G. Scalmani, V. Barone, B. Mennucci, G. A. Petersson, H. Nakatsuji, M. Caricato, X. Li, H. P. Hratchian, A. F. Izmaylov, J. Bloino, G. Zheng, J. L. Sonnenberg, M. Hada, M. Ehara, K. Toyota, R. Fukuda, J. Hasegawa, M. Ishida, T. Nakajima, Y. Honda, O. Kitao, H. Nakai, T. Vreven, J. A. Montgomery, Jr., J. E. Peralta, F. Ogliaro, M. Bearpark, J. J. Heyd, E. Brothers, K. N. Kudin, V. N. Staroverov, R. Kobayashi, J. Normand, K. Raghavachari, A. Rendell, J. C. Burant, S. S. Iyengar, J. Tomasi, M. Cossi, N. Rega, J. M. Millam, M. Klene, J. E. Knox, J. B. Cross, V. Bakken, C. Adamo, J. Jaramillo, R. Gomperts, R. E. Stratmann, O. Yazyev, A. J. Austin, R. Cammi, C. Pomelli, J. W. Ochterski, R. L. Martin, K. Morokuma, V. G. Zakrzewski, G. A. Voth, P. Salvador, J. J. Dannenberg, S. Dapprich, A. D. Daniels, Ö. Farkas, J. B. Foresman, J. V. Ortiz, J. Cioslowski, and D. J. Fox, Gaussian, Inc., Wallingford CT, 2009. 